\title{
Révision TARMED: le projet TARVISION
}

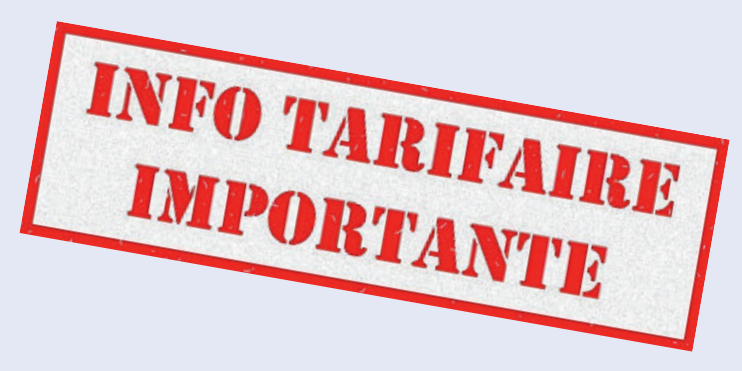

Ernst Gähler ${ }^{a}$, Roger Scherrer ${ }^{b}$

a Dr méd., vice-président de la FMH, responsable du domaine

Tarifs et Conventions

b Chef de projet TARVISION
Comme la révision urgente de la structure tarifaire du TARMED n'avance pas chez TARMED Suisse, le Comité central et l'Assemblée des délégués de la FMH ont décidé de prendre les choses en main conjointement avec l'association des hôpitaux suisses H+. La FMH a ainsi lancé le projet TARVISION lors de la journée des délégués tarifaires du 20 octobre 2010 et en a confié la direction à Roger Scherrer, un chef de projet expérimenté. En décembre 2010, la FMH a invité les sociétés de discipline à plusieurs séances d'information.

\section{Une révision tarifaire complète est-elle vraiment nécessaire?}

Le projet TARMED 2010 de TARMED Suisse est bloqué déjà depuis novembre 2008 car santésuisse exige une convention sur la régulation des coûts. Les négociations de la FMH avec santésuisse au sujet de la révision tarifaire TARMED font du surplace malgré le consensus trouvé à la rencontre au sommet du 29 avril 2010. Or il est nécessaire de procéder à cette révision de toute urgence:

- La structure tarifaire TARMED doit être remaniée et adaptés aux conditions actuelles. Les déséquilibres doivent être corrigés. Cette révision doit, entre autres, améliorer la situation des médecins de premier recours et mieux prendre en compte les prestations médicales intellectuelles.

- Dans le passé, la maintenance de la structure tarifaire n'a pas été suffisante et, de ce fait, le TARMED nécessite aujourd'hui d'importantes modifications. Le nombre élevé de propositions en suspens du côté de la Commission paritaire tarifaire (CPT) rend la gestion du tarif encore plus difficile. Par ailleurs, les assureurs ne s'en réfèrent de plus en plus souvent qu'aux coûts et veulent déjà économiser au niveau de la structure.

- L'innovation médicale est insuffisamment prise en compte dans le tarif.

- Les bases du tarif TARMED datent des années 90 .

- Les données à l'origine des modèles de coûts (location, salaires, frais d'appareils, etc.) émanent des années 1994 à 1996 et n'ont plus été adaptées depuis lors. Durant la même période, les frais annexes tels que les salaires des assistantes médicales ont augmenté d'environ 30\%.

Les points suivants justifient que la FMH effectue une révision à l'interne de la structure tarifaire TARMED:

- Une convention sur la régulation des coûts ne peut pas être acceptée sans un concept clair.
La FMH tient à améliorer la situation des médecins de premier recours, mais avec des lignes directrices claires en ce qui concerne la révision et le financement supplémentaire.

- Il faut réfuter le reproche d'un manque d'ordre au sein de la FMH au moyen d'un projet concret.

- La base de données doit montrer que la baisse linéaire des revenus des spécialistes, exigée par les assureurs pour améliorer la situation des médecins de premier recours, n'est pas praticable aux yeux de la FMH et qu'elle ne suffirait absolument pas.

\section{Comment le projet TARVISION va-t-il se dérouler?}

La révision tarifaire aura lieu en deux phases échelonnées dans le temps:

- La première phase comprend la révision des positions tarifaires les plus importantes par les sociétés de discipline médicale et leur validation par le Back Office (Service tarifaire de la FMH).

- La seconde phase sera consacrée à la révision des modèles de coûts.

Ces deux phases devraient être achevées à fin juin 2011 selon le calendrier ambitieux qui a été établi. Le tableau 1 en montre les étapes les plus importantes.

\section{Où en sommes-nous aujourd'hui?}

En décembre 2010, le domaine Tarifs et Conventions a organisé quatre séances pour informer les sociétés de discipline concernées sur le déroulement de la révision tarifaire TARMED. La première partie de la séance était consacrée aux conditions-cadres politiques actuelles et la seconde à la mise en route de la réalisation pratique.

Simultanément l'équipe du projet a élaboré des processus qui, d'une part, garantissent un travail efficace et le plus autonome possible au sein des sociétés tout en permettant à la FMH, d'autre part, de coordonner l'ensemble du projet. A cette fin, un paquet de tâches individuelles a été préparé pour chaque société impliquée. Ce paquet consiste en une liste des positions tarifaires les plus fréquentes à réviser en priorité. En outre, les sociétés peuvent demander, le cas échéant, différentes prestations de soutien au domaine Tarifs et Conventions de la FMH.

Le Back Office de la FMH est un élément essentiel $\mathrm{du}$ projet au niveau institutionnel. Ces prochains mois, il aura la tâche importante de réaliser la comparaison transversale des différentes modifications proposées par les sociétés de discipline médicale et de trouver un consensus. 


\section{Tableau 1}

Les deux phases du projet TARVISION.

\begin{tabular}{|c|c|c|c|}
\hline \multirow{2}{*}{$\begin{array}{l}\text { Activité } \\
\begin{array}{|c|}\text { Séances d'information pour } \\
\text { les sociétés de discipline (SDM) }\end{array}\end{array}$} & & Description & Calendrier \\
\hline & & $\begin{array}{l}\text { Les responsables tarifaires des SDM sont informés } \\
\text { du projet et de leurs tâches. }\end{array}$ & décembre 2010 \\
\hline $\begin{array}{c}\text { Révision des positions } \\
\text { tarifaires par les sociétés } \\
\text { de discipline }\end{array}$ & & $\begin{array}{l}20 \text { SDM mettent à jour leurs positions } \\
\text { les plus fréquentes. } \\
\text { Cette révision se concentre sur les paramètres } \\
\text { «Minutages» et «Valeur intrinsèque». }\end{array}$ & mi-avril 2011 \\
\hline \multirow[t]{2}{*}{$\begin{array}{l}\text { Validation par le Back } \\
\text { Office FMH }\end{array}$} & \multirow[b]{2}{*}{$\begin{array}{l}\text { Négociations } \\
\text { SDM - Back Office }\end{array}$} & \multirow{2}{*}{$\begin{array}{l}\text { Les modifications proposées par les SDM sont } \\
\text { examinées par des experts tarifaires du Back Office } \\
\text { de la FMH: } \\
\text { - Sont-elles correctes sur le plan } \\
\text { de la technique tarifaire? } \\
\text { - Résistent-elles à une comparaison croisée entre } \\
\text { sociétés de discipline? } \\
\text { Cela permet d'ajuster les modifications entre } \\
\text { les sociétés. }\end{array}$} & \multirow[t]{2}{*}{ mai 2011} \\
\hline & & & \\
\hline $\begin{array}{l}\text { Révision des modèles } \\
\text { de coûts }\end{array}$ & & $\begin{array}{l}\text { Mise à jour des modèles de coûts: examen } \\
\text { et actualisation des valeurs clés (salaires du personnel } \\
\text { non médical, appareils, locations, etc.). }\end{array}$ & mai 2011 \\
\hline $\begin{array}{l}\text { Nouveau calcul } \\
\text { du tarif (FMH) }\end{array}$ & & $\begin{array}{l}\text { Tous les paramètres adaptés et les nouvelles valeurs clés } \\
\text { des modèles de coûts sont introduits dans le système. } \\
\text { Le tarif fait ensuite l'objet d'un nouveau calcul. }\end{array}$ & juin 2011 \\
\hline $\begin{array}{l}\text { Négociations avec } \\
\text { les partenaires tarifaires }\end{array}$ & & $\begin{array}{l}\text { Les négociations pour définir les prix avec } \\
\text { les partenaires contractuels peuvent commencer. }\end{array}$ & $\begin{array}{l}\text { dès le mois } \\
\text { de juin } 2011\end{array}$ \\
\hline
\end{tabular}

\section{Ensemble pour une structure tarifaire TARMED réaliste}

La révision de la structure tarifaire TARMED est un grand défi pour le corps médical en général et pour les sociétés de discipline en particulier. Il s'agit en effet de synthétiser les différents intérêts et de les regrouper pour aboutir à un résultat global. Il est important que les participants ne perdent pas de vue l'ensemble du projet et ne se focalisent pas seulement sur certaines positions en particulier. Le calendrier est ambitieux et les ressources sont limitées, avant tout dans les sociétés de discipline. Au final, l'enjeu est de connaître la vérité sur les coûts et de disposer de faits clairement établis.

Les séances d'information organisées en décembre 2010 ont permis à la FMH de créer une base de confiance prometteuse. Les entretiens avec les délégués des sociétés ont montré qu'ils estimaient que cette révision était nécessaire. Simultanément, on s'est rendu compte qu'un projet complet tel que le TARVISION - avec de très nombreux acteurs et une matière très complexe - n'était réalisable qu'avec une communication intensive et ouverte dans un climat de confiance réciproque. La prochaine séance d'information importante sur le projet TARVISION aura lieu à la journée des délégués tarifaires du 23 mars 2011 à Berne. D'ici là, le domaine Tarifs et Conventions de la FMH élaborera d'autres canaux d'information de manière à pouvoir informer tous les acteurs en temps utile.

«Ensemble, on est plus efficace»: cette devise est valable aussi bien pour la collaboration de la FMH

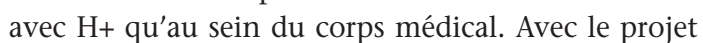
TARVISION, la FMH prouve que les médecins sont capables d'assurer leur cohésion interne et d'atteindre ensemble un objectif. 